\title{
BMJ Open Exploring healthcare providers' perspectives of the paediatric discharge process in Uganda: a qualitative exploratory study
}

\author{
Brooklyn Nemetchek, ${ }^{1}$ Asif Khowaja, ${ }^{2}$ Anthony Kavuma, ${ }^{3}$ Olive Kabajaasi, ${ }^{3}$ \\ Alex Olirus Owilli, ${ }^{1} \mathrm{~J}$ Mark Ansermino, ${ }^{4,5}$ Susan Fowler-Kerry, ${ }^{1}$ Shevin T Jacob, ${ }^{3,6}$ \\ Nathan Kenya-Mugisha, ${ }^{3}$ Jerome Kabakyenga, ${ }^{7}$ Matthew O Wiens ${ }^{\circledR 5,8}$
}

To cite: Nemetchek B, Khowaja A, Kavuma A, et al. Exploring healthcare providers' perspectives of the paediatric discharge process in Uganda: a qualitative exploratory study. BMJ Open 2019;9:e029526. doi:10.1136/ bmjopen-2019-029526

- Prepublication history and additional material for this paper are available online. To view these files, please visit the journal online (http://dx.doi org/10.1136/bmjopen-2019029526).

Received 31 January 2019 Revised 14 June 2019 Accepted 25 July 2019
Check for updates

(C) Author(s) (or their employer(s)) 2019. Re-use permitted under CC BY-NC. No commercial re-use. See rights and permissions. Published by BMJ.

For numbered affiliations see end of article.

Correspondence to Dr Matthew 0 Wiens; mowiens@outlook.com

\section{ABSTRACT}

Introduction The burden of childhood mortality continues to be born largely by low-income and middle-income countries. The critical postdischarge period has been largely neglected despite evidence that mortality rates during this period can exceed inpatient mortality rates. However, there is a paucity of data on the paediatric discharge process from the perspective of the healthcare provider. Provider perspectives may be important in the development of an improved understanding of the barriers and facilitators to improving the transition from hospital to home.

Objectives To explore healthcare providers' and facility administrators' perspectives of the paediatric discharge process with respect to: (1) current procedures, (2) barriers and challenges, (3) ideas for change, (4) facilitators for change and (5) the importance of discharge planning.

Design A qualitative exploratory approach using focus groups (14) and in-depth interviews (7).

Setting This study was conducted at seven hospitals providing paediatric care in Uganda.

Results Current discharge procedures are largely based on hospital-specific protocols or clinician opinion, as opposed to national guidelines. Some key barriers to an improved discharge process included caregiver resources and education, critical communication gaps, traditional practices, and a lack of human and physical resources. Teamwork and motivation to see improved paediatric transitions to home were identified as facilitators to implementing the ideas for change proposed by participants. The need for a standardised national policy guiding paediatric discharges, implemented through education at many levels and coupled with appropriate community referral and follow-up, was broadly perceived as essential to improving outcomes for children. Conclusions Although significant challenges and gaps were identified within the current health system, participants' ideas and the identified facilitators provide a significant basis from which change may occur. This work can facilitate the development of sustainable and effective interventions to improve postdischarge outcomes in Uganda and other similar settings.

\section{Strengths and limitations of this study}

- This study represented four regions in Uganda and included both private and public facilities.

- This study may have limited generalisability to other countries, particularly those outside of sub-Saharan Africa.

- Although some focus groups were at times small, the high degree of interest and participation provided deep insights into barriers faced by facilities, health workers and caregivers.

- The lack of the caregiver perspective limits some of the conclusions of these data.

- The information gathered through these qualitative interviews can provide critical information in designing effective interventions to improve the paediatric discharge process.

\section{BACKGROUND}

The third of 17 Sustainable Development Goals commit the world to 'ensure healthy lives and promote well-being for all at all ages' by the year 2030, a key aspect of which is decreasing under-five mortality. ${ }^{1}$ However, the burden of under-five mortality continues to be born largely by low-income and middle-income countries (LMICs), with half of the world's total under-five deaths occurring in sub-Saharan Africa alone. ${ }^{2}$ Over the past several decades, significant effort has been made to address the diagnosis and treatment provided during acute care, but care following hospital discharge has been largely neglected in research, policy and practice. ${ }^{3}$ A recent systematic review found that in LMICs, postdischarge deaths often times exceed the in-hospital mortality rate. Furthermore, most postdischarge deaths occur at home, suggesting that the point of discharge represents an important opportunity for innovation to improve health outcomes 
among children. Within the Ugandan context, a recent study found that $5 \%$ of under- 5 children who had been hospitalised for infectious illness died in the 6 months following discharge, often at home. ${ }^{4}$

Evidence from a recent proof-of-concept study in Uganda, known as Smart Discharges, demonstrated improved outcomes following discharge through an educational intervention and community-level referrals for follow-up. ${ }^{5}$ Discharge education and follow-up after discharge appear to be critical components necessary to improve the long-term survival of children admitted with serious infectious illness. ${ }^{5}$ However, little is known regarding the facilitators and barriers to adoption within the Ugandan health system. Understanding the current discharge processes in Ugandan hospitals and the challenges they face is a necessary step prior to further adoption and scaling-up of such processes. Therefore, within the context of severe paediatric infectious illness, this study aimed to explore healthcare providers' and facility administrators' perspectives of the discharge process, with respect to: (1) current procedures, (2) barriers and challenges, (3) ideas for change, (4) facilitators for change and (5) the importance of discharge planning.

\section{METHODS \\ Design}

A qualitative exploratory study was conducted and the data were prospectively collected through focus groups (FGs) and in-depth interviews with key professional stakeholders in order to explore the current paediatric discharge process within the Ugandan healthcare system, an area that has yet to be extensively studied. This study design is ideal for understanding and describing the gap in this area of research, creating a basis on which further research may build.

\section{Patient and public involvement}

Patients and public were not involved in the development of the research question or outcomes.

\section{Study setting}

The study was conducted at seven sites across Uganda, including five public, government-funded hospitals and two private not-for-profit hospitals. Government-funded hospitals included: Lira Regional Referral Hospital, Gulu Regional Referral Hospital, Jinja Regional Referral Hospital, Masaka Regional Referral Hospital and Mbarara Regional Referral Hospital. Private not-for-profit hospitals included Holy Innocents Children's Hospital in Mbarara and Kisiizi Hospital in Rukungiri district. The hospitals represent the regional distribution of major hospitals across Uganda. Hospitals varied with regard to bed capacity, number of annual paediatric admissions and number of staff (table 1).

\section{Sampling and inclusion criteria for FGs and in-depth interviews}

Site participants were recruited using purposive sampling. Front-line paediatric providers (ie, nurses and doctors) who had worked in the paediatric ward for at least 2 months preceding the time of data collection were eligible FG participants. Hospital administrators were considered eligible for participation in the in-depth interviews if they were currently involved in clinical administrative work and had some degree of oversight for the paediatric ward. Initial contact with all eligible study participants was through each respective hospital's human resources department.

\section{Data collection}

Interviews and FGs were conducted between April and July 2018. Fourteen FG discussions—-seven with nurses and seven with physicians/clinical officers-were conducted across the seven study sites, together with seven in-depth

\begin{tabular}{|c|c|c|c|c|c|}
\hline Hospital site & $\begin{array}{l}\text { Approximate total bed } \\
\text { capacity }\end{array}$ & $\begin{array}{l}\text { No of paediatric } \\
\text { beds }\end{array}$ & $\begin{array}{l}\text { No of children age } 0-5 \\
\text { years admitted annually* }\end{array}$ & $\begin{array}{l}\text { Paediatric } \\
\text { physicians }\end{array}$ & $\begin{array}{l}\text { Paediatric } \\
\text { nurses }\end{array}$ \\
\hline JRRH, Jinja & 408 & 50 & 7559 & 8 & 17 \\
\hline MRRH, Masaka & 330 & 45 & 4876 & 7 & 7 \\
\hline HICH, Mbarara & 60 & 60 & 5623 & 5 & 21 \\
\hline $\begin{array}{l}\text { Kisiizi hospital, } \\
\text { Rukungiri }\end{array}$ & 250 & 38 & 1326 & 5 & 11 \\
\hline LRRH, Lira & 346 & 70 & 3428 & 4 & 20 \\
\hline
\end{tabular}

*Average of total admissions aged 0-60 months for the 3 years: 2015, 2016 and 2017.

†Includes 9 Senior House Officers (masters of paediatric medicine students who attend to patients as part of their training requirement but are not employees of Mbarara Regional Referral Hospital).

GRRH, Gulu Regional Referral Hospital; HICH, Holy Innocents Children's Hospital; JRRH, Jinja Regional Referral Hospital; LRRH, Lira

Regional Referral Hospital; MRRH-Masaka, Masaka Regional Referral Hospital; MRRH-Mbarara, Mbarara Regional Referral Hospital. 


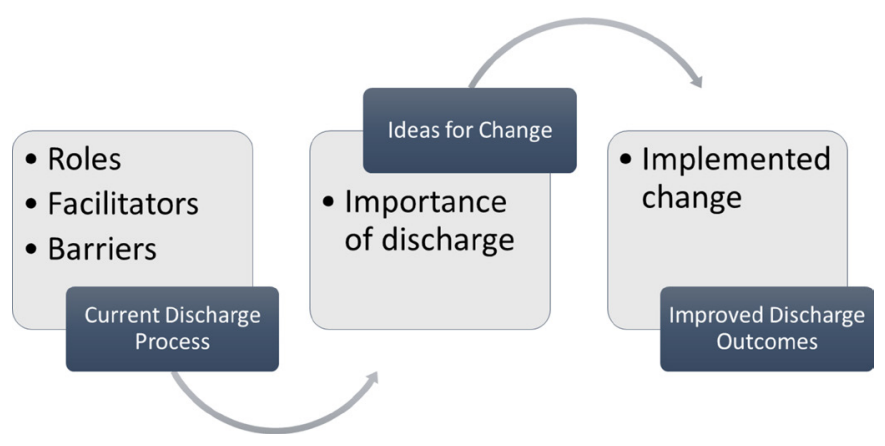

Figure 1 Conceptual framework.

interviews with hospital administrators from six study sites. The hospital administrator at one study site was unavailable during the interviewing period and was thus excluded. No other participants dropped out. Each FG consisted of 3-5 participants. Nursing FGs lasted approximately 35-75 min, clinician FGs approximately 50-80 min and in-depth semistructured interviews approximately $15-50 \mathrm{~min}$. All interviews and FGs were audio recorded following participant permission. FGs and in-depth interviews were conducted by a trained research assistant (AKa or OK) using pilot-tested semistructured interview guides consistently applied across interviews (online supplementary additional file 1). The two Ugandan interviewers, one male and one female, were hired for this specific project and had no previous involvement in Smart Discharges research or personal relationship to the study participants. Repeat interviews and participant data checking were not conducted.

\section{Ethics}

All FGs and in-depth interviews were conducted in a private hospital meeting room after obtaining written informed consent from participants. Participants were provided with an honorarium of 25,000 Ugandan Shillings (approximately US\$7).

\section{ANALYSIS}

FGs and in-depth interviews were recorded and transcribed verbatim by two interviewers (AKa and OK) and then spot checked for consistency by another member of the investigative team (BN). Transcripts were analysed using NVivo V.11 software (QST International, Cambridge, Massachusetts, USA). Mind-mapping, coding and node structures were identified and reviewed by two team members (BN and $\mathrm{AK})$. Coding of the data aimed to identify categories and linkages and to explore patterns. Relationships between five a priori themes are depicted using a conceptual framework (figure 1), from which further subthemes emerged (figure 2) in an effort to better understand the paediatric discharge process. The Standards for Reporting Qualitative Research criteria was used in reporting findings. ${ }^{6}$

\section{RESULTS}

A total of 58 participants, with wide ranges of experience and training, contributed to the 14 FGs and seven in-depth interviews (table 2). Nursing FGs included 28 nurses holding either certificates or diplomas. Nurses had, on average, more practical experience in months $(\mathrm{M}=116.7, \mathrm{SD}=90.7)$ compared with that for all participants $(\mathrm{M}=37.1, \mathrm{SD}=82.9)$. Clinician FGs included 23 medical clinicians/interns with significantly less practice experience than their nursing counterparts $(\mathrm{M}=24.1$, $\mathrm{SD}=38.2$ ). Six of the seven hospital administrators held a Masters of Medicine, with most having between 2 and 8 years of administrative experience $(\mathrm{M}=6.7, \mathrm{SD}=11.3)$.

\section{Key themes}

Five a priori themes became the framework for initial analysis, from which subthemes and concepts emerged, expanding the coding scheme to bring further clarity and understanding to the paediatric discharge process (figure 2).

\section{Current procedures}

Participants described typical daily activities in the paediatric wards, including ward rounds, seeing outpatients, admitting, treating and discharging children. Participants generally stated that they were unaware of written

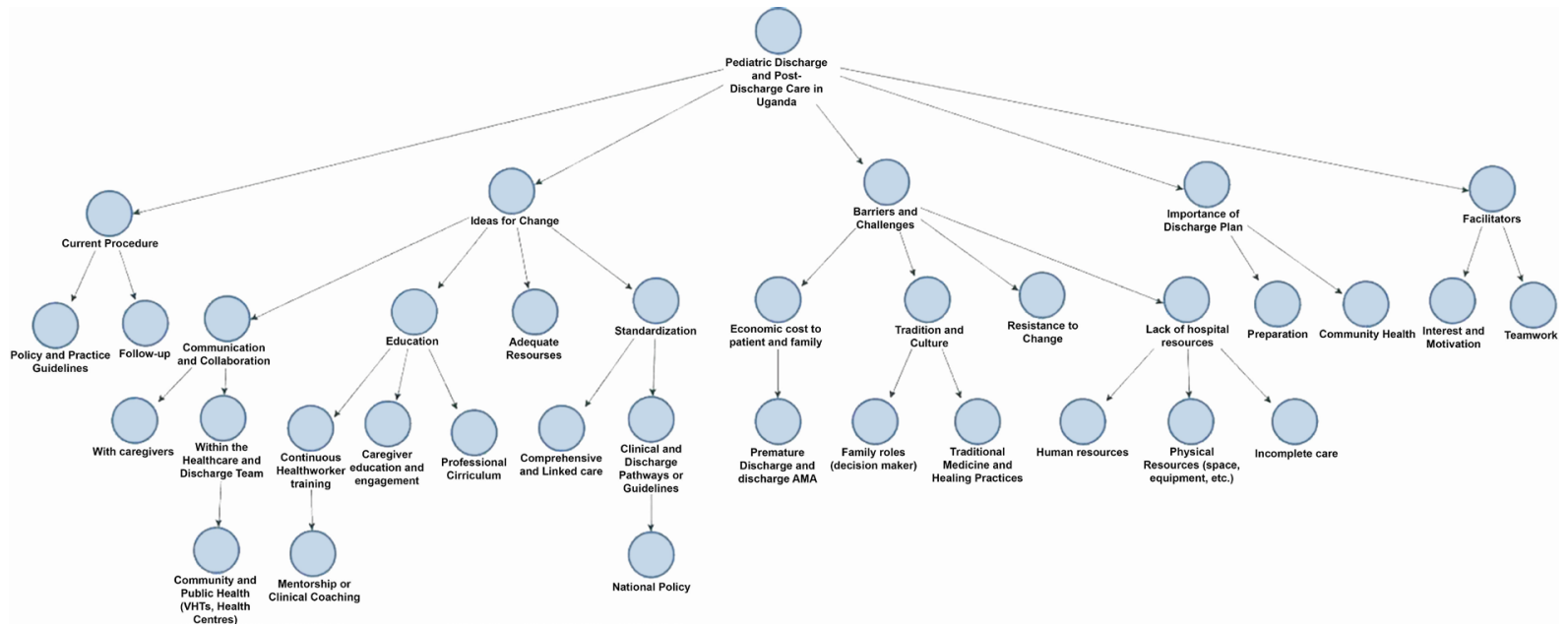

Figure 2 Discharge theme map. VHTs, village health teams. AMA, against medical advice. 
Table 2 Participant demographics

\begin{tabular}{|c|c|c|c|c|c|}
\hline Hospital & Healthcare worker & No of participants & Gender ( $\%$ female) & Age group & Education \\
\hline \multirow[t]{3}{*}{ GRRH, Gulu } & Nursing & 4 & 100 & $\begin{array}{l}1(25-33) \\
1(34-41) \\
2(42-49)\end{array}$ & $\begin{array}{l}1 \text { (certificate) } \\
3 \text { (diploma) }\end{array}$ \\
\hline & Clinician & 4 & 25 & $\begin{array}{l}1(18-25) \\
2(26-33) \\
1(42-49)\end{array}$ & $\begin{array}{l}3 \text { (intern) } \\
1 \text { (MBChB) }\end{array}$ \\
\hline & Administration & 1 & 100 & $(42-49)$ & MMed \\
\hline \multirow[t]{3}{*}{ JRRH, Jinja } & Nursing & 5 & 100 & $\begin{array}{l}3(26-33) \\
2(34-41)\end{array}$ & $\begin{array}{l}2 \text { (certificate) } \\
3 \text { (diploma) }\end{array}$ \\
\hline & Clinician & 4 & 0 & $\begin{array}{l}2(26-33) \\
2(34-41)\end{array}$ & 4 (intern) \\
\hline & Administration & 2 & 50 & $2(42-49)$ & $\begin{array}{l}1 \text { (diploma) } \\
1 \text { (MMed) }\end{array}$ \\
\hline \multirow[t]{3}{*}{ MRRH, Masaka } & Nursing & 3 & 100 & $\begin{array}{l}1(26-33) \\
1(34-41) \\
1(42-49)\end{array}$ & $\begin{array}{l}1 \text { (certificate) } \\
2 \text { (diploma) }\end{array}$ \\
\hline & Clinician & 3 & 0 & $\begin{array}{l}1(18-24) \\
1(26-33) \\
1(34-41)\end{array}$ & 3 (intern) \\
\hline & Administration & 1 & 0 & $(42-49)$ & MMed \\
\hline \multirow[t]{3}{*}{ MRRH, Mbarara } & Nursing & 4 & 100 & $\begin{array}{l}2(18-25) \\
1(26-33) \\
1(42-49)\end{array}$ & $\begin{array}{l}3 \text { (certificate) } \\
1 \text { (diploma) }\end{array}$ \\
\hline & Clinician & 4 & 75 & $\begin{array}{l}3(26-33) \\
1(34-41)\end{array}$ & 4 (MBChB) \\
\hline & Administration & 1 & 0 & $(34-41)$ & MMed \\
\hline \multirow[t]{3}{*}{$\mathrm{HICH}$, Mbarara } & Nursing & 4 & 75 & $\begin{array}{l}1(18-25) \\
3(26-33)\end{array}$ & $\begin{array}{l}2 \text { (certificate) } \\
2 \text { (diploma) }\end{array}$ \\
\hline & Clinician & 3 & 33 & $3(26-33)$ & 3 (MBChB) \\
\hline & Administration & 1 & 0 & $(34-41)$ & MMed \\
\hline \multirow[t]{3}{*}{$\begin{array}{l}\text { Kisiizi Hospital, } \\
\text { Rukungiri }\end{array}$} & Nursing & 3 & 66 & $\begin{array}{l}1(18-25) \\
1(26-33) \\
1(34-41)\end{array}$ & $\begin{array}{l}1 \text { (certificate) } \\
2 \text { (diploma) }\end{array}$ \\
\hline & Clinician & 2 & 0 & $\begin{array}{l}1(26-33) \\
1(34-41)\end{array}$ & 2 (intern) \\
\hline & Administration & 1 & 0 & $>50$ & MMed \\
\hline \multirow[t]{3}{*}{ LRRH, Lira } & Nursing & 5 & 100 & $\begin{array}{l}2(26-33) \\
1(34-41) \\
2(42-49)\end{array}$ & 5 (diploma) \\
\hline & Clinician & 3 & 33 & $3(26-33)$ & $\begin{array}{l}2 \text { (intern) } \\
1 \text { (MBChB) }\end{array}$ \\
\hline & Administration & - & - & - & - \\
\hline
\end{tabular}

GRRH, Gulu Regional Referral Hospital; HICH, Holy Innocents Children's Hospital; JRRH, Jinja Regional Referral Hospital; LRRH, Lira Regional Referral Hospital; MRRH-Masaka, Masaka Regional Referral Hospital; MRRH-Mbarara, Mbarara Regional Referral Hospital.

guidelines or policies regarding discharge processes for children admitted with infectious illnesses. Although the Uganda Clinical Guidelines $2016^{7}$ were occasionally mentioned, participants largely cited either hospital-specific standard operating procedures for the specific disease processes, implied institutional policy or departmental culture as the basis for current discharge practices. As one administrator stated, 'I think really they [standards/ guidelines] are implied, they are not explicit that they are written down, and that's where the gaps are' (Admin $5)$. These implied criteria are based on assessed clinical improvement. Health professionals in the FGs noted that mothers with more schooling had greater understanding of the importance of follow-up for their child. 
Current paediatric postdischarge follow-up procedures were described, with one administrator noting the only community-level, postdischarge follow-up that occurred was that undertaken by research studies. Clinicians at one of the private hospitals stated that one nurse travelled each Saturday to different regions in the hospital's catchment area to visit malnourished children recently discharged; however, all other children were given a follow-up date at the discharging hospitals outpatient clinic. Participants consistently identified follow-up clinics for specific chronic diseases such as HIV, tuberculosis, malnutrition, sickle cell and cardiac conditions. Referrals to these chronic diseases-specific clinics are common and often stated to be part of guidelines; however, patients admitted for acute infectious illness are given a postdischarge review date dependent on clinician judgement determined on an individual basis and highly reliant on the many compounding factors (such as severity of illness, condition at discharge, etc) involved in the child's illness. Most participants stated they did not give review dates to all children with infectious illnesses, and review dates, when given, were scheduled in an outpatient clinic (usually the discharging facility) at 2-3 weeks postdischarge.

\section{Barriers/challenges}

\section{Barrier: socioeconomic cost to patients and families}

Socioeconomic issues, such as finances and transportation, play a significant role in all aspects of health and health seeking; for discharge and follow-up care, and in timely initial treatment, readmission or referral to a higher level of care. Clinicians talked of caregivers reaching the hospital with a child too sick to save: 'They will tell you they have taken like three days because they were looking for money to meet their transport costs' (Clinician 23). Private hospital employees cited challenges related to bills incurred. Parents desperate for care bring a child to a private hospital, but, on discharge, they are unable to pay their bill or decide to forego any postdischarge treatment due to finances.

Both private and public hospitals frequently talked about discharging children prematurely or against medical advice, often due to the caregiver's request. This decision was largely related to the financial burden that families experience in caring for their hospitalised child or the need to care for other children at home. "Sometimes you want to keep the patient for a longer time but they are unable, they are unwilling to stay. So... you make a decision that is not called for and you discharge them prematurely' (Clinician 1).

The financial state of the family is also influential when a clinician recommends medication to be taken at home or that a child be followed up after discharge. Regional referral hospitals (RRHs) in Uganda are few and yet serve large catchment areas, both in terms of geography and population, making follow-up at this level of facility very difficult for patients and facilities. It is common practice to send a child home with oral antibiotics; however, medications are often not free and thus are sometimes not purchased, leaving children vulnerable to subsequent deterioration or recurrence of infection. Whether families purchase these medications is not known unless they attend a follow-up appointment or are readmitted to hospital. 'There are really mothers even if Ampiclox [antibiotic] is two thousand [Ugandan Shillings; about $\$ 0.50$ USD], they will not buy it; they don't have the money' (Clinician 21). One administrator talks of the many interwoven socioeconomic barriers to complete care for the child, saying: 'there are always issues of resources and transport... if the health facility that you want the child to be seen at is far away, then they are unlikely to go there because mostly the people we treat are very poor so they can't afford to come back or to buy the medicines or even to buy the basic things like soap for hand washing. Or they don't have access to clean water' (Admin 7).

\section{Barrier: tradition and culture}

Traditional and cultural practices and family roles are important aspects of health, including a child's discharge and follow-up. Although a mother may wish for her child to remain admitted, purchase medications or attend a follow-up appointment, pressure from the father or male decision-maker may limit her ability to complete all necessary aspects of care. Fathers often hold the finances, thus directly affecting whether the mother can complete a follow-up appointment or obtain medications on discharge. Elders (such as the mother-in-law of the mother) also influence health-seeking behaviour and may lead a caregiver to either forego formal medical care or supplement with traditional healing practices, which may itself hinder a child's recovery or even precipitate further illness and infection. Traditional healers at times conduct non-sterile surgical procedures: '...they have this pressure at home and they are like no this condition will not be treated by those medications; if we did these cuts to the child's body, they will help... like there are those cases we receive here; the child has been having diarrhoea, he is dehydrated, he is not feeding. So this, in the community there, will be interpreted as the child having 'ebiino' [false teeth] — that is why the child is not breastfeeding. Yet you as a clinician, you know that this child is dehydrated and this is why the child has failed to breastfeed' (Clinician 1). Sometimes when health workers discharge a child prematurely, they knew that it was because the family wanted to try cultural healing practices: 'these common cases of upper respiratory tract infections, from the hospital, they [go] for local tonsillectomy. One, two, three days the child is back with severe anaemia, septicaemia, very sick' (Clinician 23).

\section{Barrier: lack of hospital resources}

The issue of human resources largely equates to workload and the understaffing that is prevalent in Ugandan medical facilities. Participants talk of a nurse working alone in a ward, how she cannot do all of the procedures, administer medications and provide thorough teaching, and so, she will end up prioritising emergencies and 
each interaction may have lesser quality than she would have liked to provide. Clinicians see large numbers of children each day and may, therefore, either prioritise cases or allocate tasks to students. Many of the RRHs are teaching hospitals. One hospital stated that due to inadequate staffing, medical interns and senior house officers in paediatrics usually ran the paediatric ward. Busy hospitals mean that the most experienced are managing the emergencies, leaving junior clinicians or studentsthose least experienced-to run the wards and manage discharges.

Issues of inadequate physical resources include shortages of supplies and medications, lack of investigatory capacity, lack of sufficient beds or hospital space, among other physical constraints present in Ugandan paediatric wards. Participants from many hospitals stated that their hospital laboratory analyser for measuring complete blood counts was out of commission, meaning that even simple investigations often used to guide treatment and assess readiness for discharge had to be done from generally unaffordable private laboratory facilities: 'At discharge, most of the diagnosis we make here, we do them clinically because of lack of investigations. So, we usually discharge patients when we don't know the real focus. And at times because we are just treating generally we might not have really tackled the focus of the septicaemia and the children usually come back' (Clinician 16). Hospital bed capacity also affects inpatient care and a child's discharge, as clinicians at times are forced to discharge prematurely due to space constraints. It is common to see one bed with up to four paediatric patients, each with differing conditions, '...this one maybe has measles which has not shown up, this one has acute watery diarrhoea, then this one has... you know, we are just infecting and not helping the children' (Clinician 10).

\section{Ideas for change}

Administrators, clinicians and nurses brought forward ideas for change largely in relation to the many barriers and challenges they had identified.

\section{Idea: adequate resources}

Participants spoke to the need for adequate human and physical resources, which would improve their ability to investigate, diagnose, treat and discharge children admitted for infectious illness. It was acknowledged that families often do not purchase items such as discharge medications that are not provided outside of the public system due to their socioeconomic status. Therefore, the common perception was that public, government-funded facilities should be equipped with the necessary supplies to treat a child and provide medications required on discharge in order to ensure a full recovery. Further resources not currently in place in many facilities were suggested as potentially beneficial, such as including a social worker as part of the discharge team and a ward telephone to be used for follow-up.
Ideas: standardisation

Comprehensive, linked care was deemed necessary to improve current practices. A key aspect of comprehensive care is strengthening the Ugandan referral system, which was stated to not function optimally in its current state. Many participants envisioned a system in which, on discharge, children could be connected to their local health centre or district hospital where they could be followed up and referred back up to the RRHs if required. Referral both up and down the chain was identified to be a current difficulty in the system. 'Continuity of care from the hospital to the community; I think that is the best way we can help these children of ours grow very well' (Clinician 8). In-hospital care should not stand alone, but be integrated within a larger vision; according to participants, a holistic approach is key for lasting change and improved outcomes after discharge, including aspects of preventive and community measures such as immunisation, sanitation, clean water, education, transport and road improvement.

Clinical pathways/guidelines for discharge, at both national and hospital levels, were considered of great importance to every health provider interviewed. National policy from the Ugandan Ministry of Health and other governing bodies influence what occurs in the medical system. Clinicians stated that having a well-designed, endorsed national policy for discharge from RRHs would be essential to ensure uptake and standardisation both within and between care facilities. However, change requires political will, so '... if we don't have a lot of buy-in or commitment from the Ministry of Health and support for them because we need resources, then it becomes difficult to expect facilities to implement it' (Admin 7).

It was emphasised that clinical pathways or guidelines implemented at the national level should be applicable to the realities observed at the hospital level, taking into consideration resource availability. Participants often talked of the importance of a prestructured discharge form, wherein the discharging clinician could easily fill in all required information to provide a holistic view of the child's health status along with the comprehensive plan for discharge and follow-up. With such a standardised discharge pathway, the discharging clinician can better facilitate communication with families, the healthcare team and communities. One clinician identified the possibility of a 'discharge secretary', whose job would be to ensure that discharge forms are filled out in their entirety, complete with a clinician signature, to aid accountability that appropriate follow-up is made with lower level health facilities and to ensure that a copy of the discharge form is retained for future reference and follow-up.

\section{Idea: education}

The healthcare team bears the important role of health education: '.. to see that they will not come back in the same situation again. Should it happen again, they will go for healthcare faster than they came this time around. 
And if it happens to another child, they should be able to identify that this one is this, and it doesn't need local herbs or anything, it needs to go to the hospital' (Clinician 3). One administrator from a private hospital spoke of their hospitals move towards providing a discharge summary in the local language to facilitate communication and reinforce the education provided by health professionals. Going from individual caregiver education to the broader community level, one clinician suggested that a small compulsory course on antenatal and child health be integrated into the national education system: 'Because every woman is a potential mother even a father. So, to know certain basic, basic things, it will educate the whole nation' (Clinician 3).

A common theme among participants was that continuous health worker training is required for both those working in hospitals and those working at lower level facilities and within communities, in order to ensure all parties are informed. Whatever protocol or guideline is put into place, they must be both accessible in real time and paired with appropriate training to ensure their relevance and applicability are optimally conveyed to the end user: 'if you make the clinicians understand the contents of that template, it can also increase its acceptability and its being put to use' (Clinician 20). Once a change to a standardised discharge protocol is developed and implemented, clinicians and administrators spoke of the need to also include it into preservice training (ie, medical and nursing curricula).

Health worker education includes equipping and empowering professionals working in lower level facilities and communities to be able to receive discharged children in follow-up (down referrals) and to manage simple cases, and in so doing, take some of the burden off of RRHs. 'I would want to be able to train and coach and mentor the health workers at the lower level to be able to carry some of this' (Admin 2). At the local level, village health teams (VHTs) are members of each community who may be trained and then used in follow-up for children recently discharged, assisting the caregiver with identifying signs of further illness and reminding of the importance of follow-up appointments.

\section{Idea: communication and collaboration}

Caregivers are important members of the healthcare team; they are constantly with the child and are often used by health professionals as a source of history, presenting illness and treatment progression. 'There is this saying that the mother is the best doctor because she spends most of the time with her child' (Clinician 22). The mother or caregiver ought to participate in determining the child's readiness for discharge as she is ultimately the one who will continue to care for the child on discharge. Communicating well with the caregiver from the beginning-from admission-may facilitate an open and understanding relationship between the family and medical team and ensure that caregivers are fully informed. A discharge form given to the caregiver outlining the inpatient treatment and discharge instructions is common practice in Ugandan hospitals. The private hospitals both mentioned a gradual transition to a computerised system, which would include the retention of an electronic copy of medical records, including the discharge form. This will aid in communication and continuity of care for those patients who do not bring their discharge form for follow-up visits or readmissions.

Discharge as a team activity requires collaboration and clear communication. Key players identified in the discharge team included the caregivers, clinicians, nurses, administration and other health professionals such as consultants, nutritionists or physiotherapists involved in the care of the hospitalised patient. Although participants spoke to the unlikely nature of being able to gather together to discharge patients, this barrier was thought to be able to be mediated by strong communication within the team and fulfilling one's professional role. Clinicians stated the value of nursing staff, noting that nurses have thoughtful insights gained through the time spent assessing patients throughout their shifts.

Collaboration and communication at the community level include interfacility communication between hospitals, lower level health facilities and VHTs. Given that RRHs do not have the capacity to follow up all discharged children, follow-up is an important community-level aspect of care. Many participants thought that follow-up could be conducted by the health facility nearest the patient's home or by the VHT. An added benefit to local follow-up is that these interactions are convenient times to address broader public health issues affecting children, families and the communities at large, such as immunisation, hygiene and other areas of health promotion and preventative medicine.

\section{Facilitators}

Participants overall noted an attitude of teamwork, motivation and interest in the discharge and follow-up process as an important aspect of a child's health journey. Participants consistently referred to themselves as a team working towards the common, shared goal of healthier patients, communities, and society. '... my team, they are willing, they have that desire to make sure that their patients survive... most of them go over and above what is their call to serve those children... So I know they don't want them to go and die at home so the willingness to find a solution if they know somebody can do to keep these children alive I know they would jump on it... the teams work with that passion to keep those babies alive and grow to adulthood' (Admin 2).

\section{Importance of discharge planning}

When asked about the importance of discharge planning, participants often spoke of preparation. Discharge planning allows families and the healthcare team to prepare for a child to return to their community. 'Its like preparation for this child to get back into the community... you can get to know how to discharge this patient and how to 
help them when they get back into the community' (Nurse 22). A plan for discharge can allow the healthcare team to communicate early on with the family about a pending discharge, allowing them time to coordinate issues such as transportation or purchasing any medications required after discharge, thus ensuring the readiness of the family for the transition home. Discharge planning allows for a consistent understanding and continuous evaluation of readiness for discharge, which may help reduce premature or uncoordinated discharges that lead to deaths or frequent readmissions and associated healthcare costs to both families and government facilities. There was a recognised need to carefully manage children through to the discharge and even afterwards, which may be aided by a discharge plan. Often times for hospital staff, once a child is discharged, how a child progresses afterwards can be forgotten without something specifically guiding a process for care postdischarge.

\section{DISCUSSION}

This study found that in Ugandan hospitals, current discharge procedures for children with infectious aetiologies are largely based on hospital-specific protocol or clinician opinion, as opposed to universal guidelines. Perhaps more importantly, significant barriers to discharges are faced by facilities, healthcare staff and families, including economic costs, traditional or cultural practices, and a lack of human and physical resources. Within the context of improving the discharge process, teamwork and motivation were identified by participants as critical facilitators required for change. The need for a standardised national policy guiding paediatric discharges, implemented through education at preservice and in-service levels and coupled with appropriate community referral and follow-up, was broadly noted as essential to improving outcomes for children.

The discharge process occurs within the context of congested and overburdened facilities that are competing for resources to triage, admit, treat and discharge children. However, the challenges to providing optimal care are further compounded by traditional and cultural practices, as well as the socioeconomic status of the families of admitted children, which is consistent with previous research in Uganda examining barriers to care for children. ${ }^{8}$ The predominant cultural idea that 'traditional' and 'hospital' illnesses are mutually exclusive is often compounded with a generational conflict due to the expectation of deferring to an elder's advice. ${ }^{8}$ Financial barriers faced by families consist of transportation costs, inpatient charges at private facilities, laboratory investigations and medications prescribed on discharge, among others. These expenses are often difficult to manage, forcing families to forego continued essential care following discharge. Furthermore, with males traditionally being the decision-makers and managers of finances, inclusion of fathers in the medical care of their children may be a critical component of strategies to address postdischarge morbidity and mortality.

Clinical pathways or guidelines need to be both standardised and applicable to the reality of hospitals in LMIC settings. In the Ugandan context, current discharge criteria are majorly based on clinician assessment and facility protocol which, when described, were largely inconsistent. The ongoing Smart Discharges research program in Uganda provides a way to focus limited resources to children identified to be the most vulnerable through risk-prediction modelling. ${ }^{9}$ Using this precision public health approach, ${ }^{10}$ children identified during an admission can receive comprehensive discharge planning and guideline-based interventions. ${ }^{9}$ The same research is also developing training programmes to complement policy and guidelines, with focused training for 1) community-level health workers, 2) discharging facilities (ie, hospitals), 3) receiving facilities (ie, facilities that see children post-discharge for follow-up) and 4) the caregivers themselves. Postdischarge follow-up for the most vulnerable children is a key component of this programme. Although this study used referral hospitals, which may often be difficult for rural patients to access following discharge, the Smart Discharges programme leverages lower level facilities to conduct follow-up care through a unique 'back-referral' programme. Healthcare provider education as a key theme for change was identified by participants across this study as instrumental to ensuring robust policy development as well as integration of improved discharge practices into routine care.

Patient care is often undertaken by both professionals and students; therefore, participants identified the need for standardised, visible and implementable guidelines to help facilitate holistic paediatric care. One study analysing the sustainability of implementing guidelines for pneumonia in LMICs found that ever-changing staff played a negative role in the sustainability of interventions, emphasising the need for all levels of health professionals to be informed and well versed in the protocols, with emphasis on passing on the information to new healthcare workers in order to continue the best practice. ${ }^{11}$ An important gap in current work is the need to affect discharge practices through improved policy and guidelines at both national and hospital levels. Every FG or individual interviewee spoke to the importance of guidelines for practice. Improvement in this regard can, thus, provide the foundation for positive change in the postdischarge care of children.

Participants continually emphasised the importance of discharge planning for children throughout the interviews, expressing unity and motivation to implement process changes. An Iranian study found that a breakdown in communication between the healthcare team, patients, and families contributed to parents' decisions to discharge their children against medical advice, a potential contributor to postdischarge mortality. ${ }^{12}$ Empowering families, developing a trusting relationship with the healthcare team, and developing a plan for discharge at 
the time of admission were considered strategies towards improving health outcomes and compliance with medical care. $^{12}$

This qualitative exploratory study, designed to describe and provide a basis for further more conclusive research, is subject to several limitations. First, this study was conducted across seven hospitals in Uganda, both private not-for-profit and government. While this perspective added substantial geographical and cultural balance, this study does not capture all regions, and thus may be biassed towards the regions and cultures in which the study was conducted. As well, there may be a lack of transferability of findings to other countries or even other levels of health facilities. Related to this, four of the seven hospitals have been study sites for postdischarge epidemiology research, although were not involved in any interventions to improve post-discharge care. Although no hospital staff were involved in this prior research, an increased awareness of the perceived importance of discharge outcomes may have influenced the generalisability of the perspectives of these participants. Second, a difficulty in recruiting healthcare providers as evidenced by the, at times, small FG sizes or inability to reach hospital administration for an interview, was offset by the many FGs and large regional representation. Third, although length of interviews and FGs varied, duration of encounters was not determined by the facilitator; thus, interactions were terminated on the basis of participants having nothing further to identify or contribute. Finally, this study may be subject to bias due to the fact that investigators conducting the analysis have been involved in past postdischarge mortality research. Thus, preconceived notions about barriers, facilitators and ideas of change may have influenced the results. However, this bias may also be beneficial in terms of connecting prior research findings to the perspectives of the stakeholders being interviewed, thus ultimately benefiting the development of effective solutions.

\section{CONCLUSION}

Understanding the burden of postdischarge mortality in LMICs through the context of the discharge process has been a critical gap in the development of effective solutions to improve postdischarge outcomes. The importance of postdischarge mortality is highlighted through the improved understanding of current discharge practices and the exploration of barriers, facilitators and solutions from the perspective of Ugandan health professionals. The current discharge procedures are largely based on hospital-specific protocol or clinician opinion, and not standardised guidelines. Barriers to discharge are faced by facilities, healthcare staff and families, and include economic costs, traditional or cultural practices, and a lack of human and physical resources. Teamwork and motivation were identified as critical facilitators required for change. We identified a need for a standardised national policy coupled with appropriate community referral and follow-up and education as essential to improving outcomes for children. This work can serve to facilitate the development of sustainable and effective interventions to improve postdischarge outcomes in Uganda and other similar LMIC settings.

\section{Author affiliations}

${ }^{1}$ College of Nursing, University of Saskatchewan, Saskatoon, Saskatchewan, Canada

${ }^{2}$ School of Population and Public Health, University of British Columbia, Vancouver, British Columbia, Canada

${ }^{3}$ Walimu, Mbarara, Uganda

${ }^{4}$ Department of Anesthesiology, Pharmacology and Therapeutics, University of

British Columbia, Vancouver, British Columbia, Canada

${ }^{5}$ Center for International Child Health, BC Children's Hospital, Vancouver, British Columbia, Canada

${ }^{6}$ Department of Clinical Services, Liverpool School of Tropical Medicine, Liverpool, UK

${ }^{7}$ Maternal, Newborn, and Child Health Institute, Mbarara University of Science and Technology, Mbarara, Uganda

${ }^{8}$ Faculty of Medicine, Mbarara University of Science and Technology, Mbarara, Uganda

Contributors BN carried out data analysis and interpretation, drafted the initial manuscript, critically reviewed and revised the manuscript, approved the final manuscript as submitted, and agreed to be accountable for all aspects of the work. AKa contributed to data analysis, interpretation and drafting of initial manuscript, critically reviewed and revised the manuscript, approved the final manuscript as submitted and agreed to be accountable for all aspects of the work. AK carried out data acquisition, contributed to data interpretation, critically reviewed and revised the manuscript, approved the final manuscript as submitted, and agreed to be accountable for all aspects of the work. OK carried out data acquisition, contributed to data interpretation, critically reviewed and revised the manuscript, approved the final manuscript as submitted and agreed to be accountable for all aspects of the work. A00 contributed to study design and data interpretation, critically reviewed and revised the manuscript, approved the final manuscript as submitted and agreed to be accountable for all aspects of the work. JMA contributed to data interpretation, critically reviewed and revised the manuscript, approved the final manuscript as submitted and agreed to be accountable for all aspects of the work. SF-K contributed to data interpretation, critically reviewed and revised the manuscript, approved the final manuscript as submitted and agreed to be accountable for all aspects of the work. STJ contributed to data interpretation, critically reviewed and revised the manuscript, approved the final manuscript as submitted, and agrees to be accountable for all aspects of the work. NK-M contributed to study design and data interpretation, critically reviewed and revised the manuscript, approved the final manuscript as submitted and agreed to be accountable for all aspects of the work. JK contributed to study design and data interpretation, critically reviewed and revised the manuscript, approved the final manuscript as submitted and agreed to be accountable for all aspects of the work. MOW contributed to study conceptualisation and design, coordinated and supervised the project, contributed to data interpretation and drafting of initial manuscript, critically reviewed and revised the manuscript and approved the final manuscript as submitted, and agreed to be accountable for all aspects of the work.

Funding This research was funded by Grand Challenges Canada.

Competing interests None declared.

Patient consent for publication Not required.

Ethics approval Ethical approval was obtained from the research ethics boards at the University of British Columbia (H18-00403) and Mbarara University of Science and Technology (MUREC 1/7).

Provenance and peer review Not commissioned; externally peer reviewed.

Data availability statement Data are available on reasonable request.

Open access This is an open access article distributed in accordance with the Creative Commons Attribution Non Commercial (CC BY-NC 4.0) license, which permits others to distribute, remix, adapt, build upon this work non-commercially, and license their derivative works on different terms, provided the original work is properly cited, appropriate credit is given, any changes made indicated, and the use is non-commercial. See: http://creativecommons.org/licenses/by-nc/4.0/. 


\section{REFERENCES}

1. United Nations. Transforming our world: the 2030 agenda for sustainable development, 2015.

2. United Nations. The millennium development goals report. New York, 2015.

3. Nemetchek B, English L, Kissoon N, et al. Pediatric post-discharge mortality in developing countries: a systematic review. BMJ Open. In Press;2018.

4. Wiens MO, Kumbakumba E, Larson CP, et al. Postdischarge mortality in children with acute infectious diseases: derivation of postdischarge mortality prediction models. BMJ Open 2015;5:e009449.

5. Wiens MO, Kumbakumba E, Larson CP, et al. Scheduled follow-up referrals and simple prevention kits including counseling to improve post-discharge outcomes among children in Uganda: a proof-ofconcept study. Global Health: Science and Practice 2016;4:422-34.

6. O'Brien BC, Harris IB, Beckman TJ, et al. Standards for reporting qualitative research: a synthesis of recommendations. Academic Medicine 2014;89:1245-51.
7. Ministry of Health Uganda. Uganda clinical guidelines 2016. National guidelines for management of common conditions, 2016.

8. Sundararajan R, Mwanga-Amumpaire J, Adrama $\mathrm{H}$, et al. Sociocultural and structural factors contributing to delays in treatment for children with severe malaria: a qualitative study in southwestern Uganda. Am J Trop Med Hyg 2015;92:933-40.

9. Wiens MO, Kissoon N, Kabakyenga J. Smart Hospital discharges to address a neglected epidemic in sepsis in low- and middle-income countries. JAMA Pediatr 2018;172:213.

10. Dowell SF, Blazes D, Desmond-Hellmann S. Four steps to precision public health. Nature 2016;540:189-91.

11. Gray A, Chhor L, Sanyalack S, et al. Some sustained improvements in pneumonia case management four and five years following implementation of paediatric Hospital guidelines in Lao PDR. Sci Rep 2017;7:1-8.

12. Alireza NN, Hamid P, Jamalodin B. The process of parents' decisionmaking to discharge their child against medical advice (Dama): a grounded theory study. International Journal of Medical Research \& Health Sciences 2016;5:335-43. 